\title{
GEOMETRIC QUANTITIES ASSOCIATED TO DIFFERENTIAL OPERATORS
}

\author{
PIERRE MATHONET
}

\begin{abstract}
Denote by $\mathcal{F}_{\lambda}$ the space of fields of tensor densities of weight $-\lambda$ over a manifold $M$. The space $\mathcal{D}_{\lambda, \mu}^{p}$ of differential operators of order at most $p$ that map $\mathcal{F}_{\lambda}$ onto $\mathcal{F}_{\mu}$ are modules over the Lie algebra of vector fields $\operatorname{Vect}(M)$. We compute all the $\operatorname{Vect}(M)$-invariant mappings from $\mathcal{D}_{\lambda, \mu}^{p}$ onto $\mathcal{F}_{\nu}$.
\end{abstract}

\section{INTRODUCTION}

Let $M$ be a smooth, connected, Hausdorff and second countable manifold of dimension $m$. The space $\Delta^{\lambda}(M)$ of tensor densities of degree $\lambda \in \mathbb{R}$ over $M$ is the space of applications of degree $\lambda$ from $\wedge^{m} T M \backslash\{0\}$ to $\mathbb{R}$, i.e. the space of applications

$$
F:\left.\wedge^{m} T M \rightarrow \mathbb{R}|F(s \omega)=| s\right|^{\lambda} F(\omega)
$$

for any $s \in \mathbb{R} \backslash\{0\}$ and any $\omega \in \wedge^{m} T M \backslash\{0\}$. We denote by $\mathcal{F}_{\lambda}$ the space of smooth sections of $\Delta^{-\lambda}(M)$. This is a module over the Lie algebra of vector fields $\operatorname{Vect}(M)$ : over any chart $\left(U,\left(x^{1}, \ldots, x^{m}\right)\right)$, any element of $\mathcal{F}_{\lambda}$ has the form (with the standard notation)

$$
\left.F\right|_{U}=f\left|d x^{1} \wedge \ldots \wedge d x^{m}\right|^{-\lambda}
$$

where $f: U \rightarrow \mathbb{R}$ is a smooth function.

The corresponding local expression of the Lie derivative is

$$
\left.L_{X}^{\lambda} F\right|_{U}=\left(X . f-\lambda \operatorname{tr}\left(D_{x} X\right) f\right)\left|d x^{1} \wedge \ldots \wedge d x^{m}\right|^{-\lambda},
$$

where $X . f$ is the differentiation of $f$ in the direction of $X$, tr denotes the trace and $D_{x} X$ is the differential of the local expression of the vector field $X$.

By $\mathcal{D}_{\lambda, \mu}^{p}$ we denote the space of differential operators of order at most $p$ that map $\mathcal{F}_{\lambda}$ onto $\mathcal{F}_{\mu}$. This is a module over the Lie algebra of vevctor fields $\operatorname{Vect}(M)$ with respect to the Lie derivative given by the commutator

$$
L_{X}^{\lambda, \mu} D=L_{X}^{\mu} \circ D-D \circ L_{X}^{\lambda}
$$

for any $X \in \operatorname{Vect}(M)$ and $D \in \mathcal{D}_{\lambda, \mu}^{p}$. The classification of these modules was obtained in a series of recent papers $([3],[6],[5],[9]$, and [11]). Some of them were studied by the classics, especially for a manifold of dimension one (see for instance [16]). Let us quote the module $\mathcal{D}_{\frac{p-1}{2}, \frac{-p-1}{2}}^{p}$, over the circle, with particular operators of the form

$$
c D_{t}^{p}+\sum_{k=0}^{p-2} a_{k} D_{t}^{k},
$$

where $c$ is a constant. 
These operators appear in the works of Gel'fand and Dickey for the construction of the KdV hierarchies. For $p=2$, such operators are known as Sturm-Liouville operators and appear in the works of Kirillov and Segal (see [7] and [14]) as the coadjoint representation of the Virasoro algebra. The vector space of operators such as (1) is a submodule of $\mathcal{D}_{\frac{p-1}{2}, \frac{-p-1}{2}}^{p}$ for the action of the group $\operatorname{Diff}^{+}\left(S^{1}\right)$ (the group of orientation preserving diffeomorphisms over the circle) and for the action of the Lie algebra of vector fields $\operatorname{Vect}\left(S^{1}\right)$.

We will show that these submodules are the intersection of the kernels of some $\operatorname{Vect}\left(S^{1}\right)$-invariant mappings from $\mathcal{D}_{\frac{p-1}{2}, \frac{-p-1}{2}}^{p}$ into some spaces of densities $\mathcal{F}_{\nu}$. This is why we will determine any such mappings, i.e. the mappings

$$
\mathcal{T}: \mathcal{D}_{\lambda, \mu}^{p} \rightarrow \mathcal{F}_{\mu}
$$

such that

$$
L_{X}^{\nu}(\mathcal{T}(D))=\mathcal{T}\left(L_{X}^{\lambda, \mu} D\right)
$$

This will allow us to exhibit some submodules such as the space of operators (1) (by considering the kernels of the mappings $\mathcal{T}$ ), and to associate some geometric information to differential operators (by considering the image of the mappings $\mathcal{T}$ ).

\section{Multidimensional MANiFOLDS}

Invariant mappings from $\mathcal{D}_{\lambda, \mu}^{p}$ to $\mathcal{F}_{\nu}$ are particular cases of intertwining operators from $\mathcal{D}_{\lambda_{1}, \mu_{1}}^{p}$ to $\mathcal{D}_{\lambda_{2}, \mu_{2}}^{q}$. Such mappings were classified in [11] for a manifold of dimension at least two. In this section, we will recall the results of that paper.

\subsection{Some invariant mappings.}

2.1.1. The projection on the part of order zero. When $\lambda=0$, the part of order zero of an element in $\mathcal{D}_{\lambda, \mu}^{p}$ has an intrinsic meaning : it is the result of its evaluation on the constant function $1\left(\mathcal{F}_{0}\right.$ is indeed isomorphic to $\left.C^{\infty}(M)\right)$. We denote this mapping by $\mathcal{P}_{0}$ :

$$
\mathcal{P}_{0}: \mathcal{D}_{0, \mu}^{p} \rightarrow \mathcal{F}_{\mu}: D \mapsto D(1)
$$

This mapping is clearly invariant.

2.1.2. The conjugation of differential operators. The conjugation operator $\mathcal{C}$ is an isomorphism between the modules $\mathcal{D}_{\lambda, \mu}^{p}$ and $\mathcal{D}_{-1-\mu,-1-\lambda}^{p}$. It is defined by the equation

$$
\int_{M} D(f) g=\int_{M} f(\mathcal{C}(D))(g),
$$

for any $D \in \mathcal{D}_{\lambda, \mu}^{p}$, and any $f \in \mathcal{F}_{\lambda}, g \in \mathcal{F}_{-1-\mu}$, with compact support in $M$. 


\subsection{Classification of the invariant mappings.}

2.2.1. Local invariants. Here is the list of invariant mappings $\mathcal{T}$ from $\mathcal{D}_{\lambda, \mu}^{p}$ to $\mathcal{F}_{\nu}$ that preserve the support of their arguments, over a manifold of dimension at least two :

Theorem 2.1. - For any $p \geqslant 2$, the spaces of invariant mappings from $\mathcal{D}_{\lambda, \mu}^{p}$ to $\mathcal{F}_{\nu}$ are generated by the mappings

$$
\mathcal{P}_{0}: \mathcal{D}_{0, \mu}^{p} \rightarrow \mathcal{F}_{\mu}
$$

and

$$
\mathcal{P}_{0} \circ \mathcal{C}: \mathcal{D}_{\lambda,-1}^{p} \rightarrow \mathcal{F}_{-1-\lambda}
$$

- If $(\lambda, \mu) \neq(0,-1)$, there exists a one-dimensional space of invariant mappings from $\mathcal{D}_{\lambda, \mu}^{1}$ to $\mathcal{F}_{\mu-\lambda}$, generated by the mapping

$$
\mathcal{T}: \mathcal{D}_{\lambda, \mu}^{1} \rightarrow \mathcal{F}_{\mu-\lambda}: \sum_{k=0}^{m} f_{k} D_{x^{k}}+f_{0} \mapsto \lambda \sum_{k=0}^{m} D_{x^{k}} f_{k}+(\mu-\lambda+1) f_{0} .
$$

The two-dimensional space of invariant mappings form $\mathcal{D}_{0,-1}^{1}$ to $\mathcal{F}_{-1}$ is generated by $\mathcal{P}_{0}$ and $\mathcal{P}_{0} \circ \mathcal{C}$.

- The modules $\mathcal{D}_{\lambda, \mu}^{0}$ are isomorphic to $\mathcal{F}_{\mu-\lambda}$. The isomorphism is obtained by considering a density as the operator "multiplication by this density" (a kind of identity). The invariant mappings of this case are scalar multiples of this isomorphism.

2.2.2. Non local invariants. Here is the list of the invariants that do not preserve the support of their arguments, over a manifold of dimension at least two. They only exist if the manifold is compact.

Theorem 2.2. If $M$ is a multidimensional compact manifold, the space of invariant mappings $\mathcal{T}$ from $\mathcal{D}_{\lambda, \mu}^{p}$ to $\mathcal{F}_{\nu}$ is generated by the mappings

$$
\mathcal{T}_{n l, 1}: \mathcal{D}_{\lambda, \lambda-1}^{0} \cong \mathcal{F}_{-1} \rightarrow \mathcal{F}_{0}: D \mapsto \int_{M} D
$$

and

$$
\mathcal{T}_{n l, 2}: \mathcal{D}_{0,-1}^{p} \rightarrow \mathcal{F}_{0}: D \mapsto \int_{M} \mathcal{P}_{0}(D)
$$

\section{MANifoldS OF DIMENSION ONE : LOCAL INVARIANTS}

3.1. Polynomials and differential operators. The formalism that we will use for our computations was developed in [9] and already used in [11]. It is the formalism of associated polynomials. We will recall in this section how on can associate a polynomial to a differential operator over any chart domain.

Let $D$ be a differential operator of order at most $p$ between the smooth sections of two vector bundles $E_{1}(M)$ and $E_{2}(M)$ over $M$. In local coordinates on $M$ and simultaneous trivializations, $D$ has a local expression of the form

$$
D(f)_{x}=\sum_{|\alpha| \leqslant p} A_{\alpha, x}\left(D_{x}^{\alpha} f\right),
$$

where $\alpha=\left(\alpha^{1}, \ldots, \alpha^{m}\right) \in \mathbb{N}^{m}$ and $D_{x}^{\alpha} f$ denotes the partial derivative of order $\alpha$

$$
D_{x^{1}}^{\alpha^{1}} \ldots D_{x^{m}}^{\alpha^{m}} f
$$


of the local expression $f$ of the argument of $D$. Moreover the smooth map $x \mapsto A_{\alpha, x}$ takes values in the space of linear mappings between the typical fibres of the bundles.

We define the associated polynomial in the following way :

$$
P(\xi ; f)_{x}=\sum_{|\alpha| \leqslant p} A_{\alpha, x}(f) \xi^{\alpha}
$$

where $\xi_{1}, \ldots, \xi_{m}$ are the components of $\xi \in T_{x}^{*} M$ in the basis $d x^{1}, \ldots, d x^{m}$ and

$$
\xi^{\alpha}=\xi_{1}^{\alpha^{1}} \ldots \xi_{m}^{\alpha^{m}}
$$

Remark that the polynomial has values in the space of linear applications between the typical fibres of the considered bundles : $P$ is a smooth section of

$$
\vee T U \otimes \operatorname{Hom}\left(E_{1}, E_{2}\right),
$$

where $E_{1}$ and $E_{2}$ are the typlical fibres of the bundles $E_{1}(M)$ and $E_{2}(M)$. This construction extends to multidifferential operators : we then have polynomials of several variables (each variable being associated to one argument and representing the derivatives acting on this argument). In this case the polynomials have values in the space of multilinear applications between the typical fibres.

3.2. Local invariants and polynomials. It is well known (see [12]) that the restriction of local maps to any relatively compact chart domain are differential operators. Hence we can associate a polynomial to any local map such as $\mathcal{T}$ and translate the invariance equation (2) in the framework of associated polynomials : the polynomial $T$ associated to any map $\mathcal{T}$ from $\mathcal{D}_{\lambda, \mu}^{p}$ to $\mathcal{F}_{\nu}$ over a chart $(U, t)$ has values in the space of linear mappings between the typical fibres of the considered bundles. In our situation, $T$ is a section of the bundle

$$
\vee T U \otimes \operatorname{Hom}\left(\vee^{\leqslant p} T U \otimes \Delta^{\lambda-\mu}(U), \Delta^{-\nu}(U)\right),
$$

that is,

$$
\vee T U \otimes \Delta^{\mu-\lambda-\nu}(U) \otimes \operatorname{Hom}\left(\vee^{\leqslant p} T U, \mathbb{R}\right)
$$

We use the 1 -form $\theta$ to represent the derivatives acting on the components of the field $X$ in the invariance equation. Doing so, we get

$$
\begin{aligned}
\langle X, \eta\rangle(T(\eta+ & \theta, P)-T(\eta, P))-T\left(\eta+\theta, X \tau_{\theta} P\right)+\nu\langle X, \theta\rangle T(\eta, P) \\
& +(\lambda-\mu)\langle X, \theta\rangle T(\eta+\theta, P)+\lambda\langle X, \theta\rangle T\left(\eta+\theta, \tau_{\theta} P\right)-X . T=0
\end{aligned}
$$

We will use this equation for vector fields that are polynomials of degree less than or equal to three in the coordinate. It is clear that it is sufficient to get the invariance for the entire algebra of vector fields over the chart. The invariance for the fields $D_{t}$ and $t D_{t}$ leads to the following result.

Lemma 3.1. Every invariant operator from $\mathcal{D}_{\lambda, \mu}^{p}$ to $\mathcal{F}_{\nu}$ has constant coefficients over any chart domain and each associated polynomial $T\left(\eta, D_{t}^{r}\right)$ is homogeneous of degree $r+\mu-\lambda-\nu$ in $\eta$. In particular, such a polynomial has to vanish if $r+\mu-\lambda-\nu$ is not a positive integer.

Proof. Considering the degrees zero and one in $\theta$ of the invariance equation (3) leads to the system

$$
\left\{\begin{array}{lll}
X . T & = & 0 \\
\eta D_{\eta} T+(\lambda-\mu+\nu-r) T & = & 0 .
\end{array}\right.
$$

This directly yields the result. 
Definition 3.1. The shift of the modules $\mathcal{D}_{\lambda, \mu}^{p}$ and $\mathcal{F}_{\nu}$ is the real number

$$
s=\mu-\lambda-\nu .
$$

We will now express the remaining invariance equations in terms of the polynomials $T_{r}=T\left(\eta, D_{t}^{r}\right)$. We know by the previous theorem that $T_{r}$ can be written

$$
T_{r}=C_{r} \eta^{r+s}
$$

where $C_{r}$ is a constant. Hence, Equation (3) is equivalent to the system

$$
\begin{cases}(r+s)(2 \lambda-2 \mu+s-r-1) C_{r}+r(2 \lambda-r+1) C_{r-1} & =0 \\ (r+s)(r+s-1)(3 \lambda-3 \mu-2 r+s-2) C_{r} & \\ +3 r(2 \lambda-r+1)(r+s-1) C_{r-1}+r(r-1)(3 \lambda-r+2) C_{r-2} & =0 .\end{cases}
$$

The first equation is available for $r+s \geqslant 1$ and the second one is available for $r+s \geqslant 2$. The constants that are not defined in the system are set to zero (for example, the first equation is available for $r=0$ if $s$ is positive. In this case, $C_{r-1}$ is set to zero).

3.3. Constructing new invariants from old ones. Given an invariant mapping $\mathcal{T}$ from $\mathcal{D}_{\lambda, \mu}^{p}$ to $\mathcal{F}_{\nu}$, there are two situations where we can easily construct a new invariant. For the first construction, one has to consider the de Rham differential. It acts from the space $\mathcal{F}_{0}$ (which is actually isomorphic to $C^{\infty}(M)$ ) to the space $\mathcal{F}_{-1}$ (which is isomorphic to the space of one-forms, over a manifold of dimension one). The construction is the following.

Proposition 3.1. If $\mathcal{T}$ is an invariant mapping from $\mathcal{D}_{\lambda, \mu}^{p}$ to $\mathcal{F}_{0}$, then a new invariant mapping with values in $\mathcal{F}_{-1}$ is given by $d \circ \mathcal{T}$.

For the second construction, we have to consider the symmetry of the modules $\mathcal{D}_{\lambda, \mu}^{p}$ given by the conjugation, when $\lambda+\mu+1=0$ : it allows us to define an invariant projection.

Definition 3.2. If $\lambda+\mu+1=0$, we define

$$
\mathcal{P}_{\mathcal{C}}: \mathcal{D}_{\lambda, \mu}^{p} \rightarrow \mathcal{D}_{\lambda, \mu}^{p-1}: D \mapsto \frac{1}{2}\left(D-(-1)^{p} \mathcal{C}(D)\right) .
$$

The operator $\mathcal{P}_{\mathcal{C}}(D)$ has order at most $p-1$ since $\sigma_{\mathcal{C}(D)}=(-1)^{p} \sigma_{D}(\sigma$ denotes the "principal symbol" operator). Now we have

Proposition 3.2. If $\lambda+\mu+1=0$ and $\mathcal{T}$ is an invariant mapping from $\mathcal{D}_{\lambda, \mu}^{p}$ to $\mathcal{F}_{\nu}$, then a new invariant mapping is given by $\mathcal{T} \circ \mathcal{P}_{\mathcal{C}}$ from $\mathcal{D}_{\lambda, \mu}^{p+1}$ to $\mathcal{F}_{\nu}$.

Now we are able to present and discuss the invariant mappings from $\mathcal{D}_{\lambda, \mu}^{p}$ to $\mathcal{F}_{\nu}$. We will start with the minimal value for $s$ (that is $s=-p$ ) corresponding to the case $\nu=\mu-\lambda+p$, then we will go on with $s$ going from $-p+1$ to $-p+3$ and then find all the solutions for $s \geqslant-p+4$.

In each case, we will give a basis of the vector space of invariant mappings.

3.4. First invariants. The first value of $\nu$ we will consider for fixed $p, \lambda$ and $\mu$ is $\nu=\mu-\lambda+p$.

Theorem 3.1. For every $p$, the space of invariant operators from $\mathcal{D}_{\lambda, \mu}^{p}$ to $\mathcal{F}_{\mu-\lambda+p}$ has dimension one. It is generated by the principal symbol operator:

$$
\sigma: \mathcal{D}_{\lambda, \mu}^{p} \rightarrow \mathcal{F}_{\mu-\lambda+p}
$$


Proof. We have $s=-p$. Hence, only $T_{p}$ can exist : we write $T_{p}=C_{p}$ and $T_{r}=0$, for every $r \in\{0, \ldots, p-1\}$, and there is no more condition on $C_{p}$.

In local coordinates, this operator is given by

$$
\sigma\left(\sum_{k=0}^{p} f_{k} D_{t}^{k}\right)=f_{p}
$$

Next we go on with $\nu=\mu-\lambda+p-1$.

Theorem 3.2. • If $p \neq 0$ and $(\lambda, \mu) \neq\left(\frac{p-1}{2}, \frac{-(p+1)}{2}\right)$, the space of invariant operators from $\mathcal{D}_{\lambda, \mu}^{p}$ to $\mathcal{F}_{\mu-\lambda+p-1}$ has dimension one.

- If $p \neq 0$ and $(\lambda, \mu)=\left(\frac{p-1}{2}, \frac{-(p+1)}{2}\right)$, the space of invariant operators has dimension two and the operators have values in $\mathcal{F}_{-1}$.

If $p=0$, there exists a one-dimensional space of invariant operators from $\mathcal{D}_{\lambda, \mu}^{0}$ to $\mathcal{F}_{-1}$ if and only if $\mu-\lambda=0$ : this space is generated by the de Rham differential.

Proof. We have $s=1-p$. Hence, only $T_{p}$ and $T_{p-1}$ can exist. Moreover $T_{p-1}$ exists only if $p \geqslant 1$.

If $p \neq 0$, we write $T_{p}=C_{p} \eta, T_{p-1}=C_{p-1}$ and $T_{r}=0$ for every $r \in\{0, \ldots, p-2\}$. We have the following condition on the coefficients :

$$
2(\lambda-\mu-p) C_{p}+p(2 \lambda-p+1) C_{p-1}=0 .
$$

If $p=0$, the coefficient $C_{p-1}$ disappears and the condition becomes

$$
(\lambda-\mu) C_{p}=0 .
$$

This yields the result.

\section{Discussion :}

- For the "Gel'fand-Dickey" module $\mathcal{D}_{\frac{p-1}{2}, \frac{-p-1}{2}}^{p}$, a first generator is obtained by composing the de Rham differential with the operator $\sigma$ :

$$
\mathcal{T}_{\mathrm{GD}, 1}=d \circ \sigma .
$$

The second one is obtained by composing the map $\sigma$ with $\mathcal{P}_{\mathcal{C}}$ :

$$
\mathcal{T}_{\mathrm{GD}, 2}=\sigma \circ \mathcal{P}_{\mathcal{C}} .
$$

- If $p=0$ and $\mu-\lambda=0$, the module $\mathcal{D}_{\lambda, \mu}^{0}$ is isomorphic to the space $\mathcal{F}_{0}$ and the natural operator in that context is the de Rham differential. Note that this operator can be seen as a particular case of $\mathcal{T}_{\mathrm{GD}, 1}$.

In local coordinates, we have

$$
\begin{aligned}
& \mathcal{T}_{p, 1}\left(\sum_{k=0}^{p} f_{k} D_{t}^{k}\right)=p(2 \lambda-p+1) D_{t} f_{p}-2(\lambda-\mu-p) f_{p-1} \\
& \mathcal{T}_{\mathrm{GD}, 1}\left(\sum_{k=0}^{p} f_{k} D_{t}^{k}\right)=D_{t} f_{p} \\
& \mathcal{T}_{\mathrm{GD}, 2}\left(\sum_{k=0}^{p} f_{k} D_{t}^{k}\right)=f_{p-1}-\frac{p}{2} D_{t} f_{p} .
\end{aligned}
$$

Remark : The intersection of the kernels of the mappings $\mathcal{T}_{\mathrm{GD}, 1}$ and $\mathcal{T}_{\mathrm{GD}, 2}$ is constituted by the operators of the form $c D_{t}^{p}+\sum_{k=0}^{p-2} f_{k} D_{t}^{k}$, that we mentioned in the introduction.

Now, let us consider the case $\nu=\mu-\lambda+p-2$. 
Definition 3.3. We denote by $H_{p}$ the hyperbola in the space of parameters $\lambda, \mu$, defined by the equation

$$
\left(\lambda-\frac{p-2}{3}\right)\left(\mu+\frac{p+1}{3}\right)+\frac{1}{36}(p+1)(p-2)=0 .
$$

This hyperbola reduces in the case $p=3$ to the one found by H. Gargoubi in his work of classification in [5]. In the case $p=2$, it degenerates in the lines of equations $\lambda=0$ and $\mu=-1$.

Theorem 3.3. - If $p \geqslant 3$, there exists a unique (up to a constant) invariant mapping $\mathcal{T}_{p, 2}$ from $\mathcal{D}_{\lambda, \mu}^{p}$ to $\mathcal{F}_{\mu-\lambda+p-2}$ for any $(\lambda, \mu) \in H_{p}$.

- If $p=2$, the space of invariant mappings defined on $H_{2}$ is generated by $\mathcal{P}_{0}$ on the line of equation $\lambda=0$ and by $\mathcal{P}_{0} \circ \mathcal{C}$ on the line of equation $\mu=-1$.

- For $p \geqslant 1$, the scalar multiples of the operator $d \circ \mathcal{T}_{p, 1}$ are invariant mappings from $\mathcal{D}_{\lambda, \mu}^{p}$ to $\mathcal{F}_{\mu-\lambda+p-2}$ for any $(\lambda, \mu)$ such that $\mu-\lambda+p-1=0$.

Proof. We have $s=-p+2$, hence the polynomials that do not vanish are $T_{p}, T_{p-1}$ and $T_{p-2}$ if $p \geqslant 2$.

In this case we can write $T_{p}=C_{p} \eta^{2}, T_{p-1}=C_{p-1} \eta, T_{p-2}=C_{p-2}$ and $T_{r}=0$ for every $r \in\{0, \ldots, p-3\}$. Moreover, the coefficients are submitted to the system of equations

$$
\begin{cases}2(2 \lambda-2 \mu-2 p+1) C_{p}+p(2 \lambda-p+1) C_{p-1} & =0 \\ 6(\lambda-\mu-p) C_{p}+3 p(2 \lambda-p+1) C_{p-1}+p(p-1)(3 \lambda-p+2) C_{p-2} & =0 \\ 2(\lambda-\mu-p+1) C_{p-1}+(p-1)(2 \lambda-p+2) C_{p-2} & =\end{cases}
$$

We have to compute the rank of this system of equations. We then compute its determinant and get

$$
12(\lambda-\mu-p+1)(p-1)\left[\left(\lambda-\frac{p-2}{3}\right)\left(\mu+\frac{p+1}{3}\right)+\frac{1}{36}(p+1)(p-2)\right] .
$$

- For $p=2$, it is easily seen that the solutions of the system on the lines of equation $\lambda=0$ and $\mu=-1$ correspond to scalar multiples of $\mathcal{P}_{0}$ and $\mathcal{P}_{0} \circ \mathcal{C}$.

- On the hyperbola $H_{p}$ for $p \geqslant 3$, the unique (up to a multiplicative constant) solution $\mathcal{T}_{p, 2}$ corresponds to the coefficients

$$
\left\{\begin{array}{l}
C_{p}=\frac{2}{3} p(p-1)(p-3 \lambda-2)^{2} \\
C_{p-1}=2(p-1)(p-3 \lambda-2)(2+2 \lambda-p) \\
C_{p-2}=3 p^{2}-12 \lambda p+12 \lambda^{2}-11 p+24 \lambda+10
\end{array}\right.
$$

In local coordinates, we have

$$
\mathcal{T}_{p, 2}\left(\sum_{k=0}^{p} f_{k} D_{t}^{k}\right)=C_{p} D_{t}^{2} f_{p}+C_{p-1} D_{t} f_{p-1}+C_{p-2} f_{p-2} .
$$

- For $\lambda-\mu-p+1=0$ (or $\nu=-1$ ), the solution of the system is also unique (up to a constant) : it is the operator $d \circ \mathcal{T}_{p, 1}$.

- For $p=1$, the coefficient $C_{p-2}$ disappears and the coefficient $C_{1}$ and $C_{0}$ are submitted to the following conditions.

$$
\left\{\begin{array}{lll}
(2 \lambda-2 \mu-1) C_{1}+\lambda C_{0} & = & 0 \\
(\lambda-\mu-1) C_{1}+\lambda C_{0} & = & 0 \\
2(\lambda-\mu) C_{0} & = & 0
\end{array}\right.
$$

There exists a one-dimensional space of solutions if and only if $\lambda-\mu=0$. This space is generated by $d \circ \mathcal{T}_{p, 1}$. 
- For $p=0$, the coefficients $C_{p-1}$ and $C_{p-2}$ disappear and there is no solution for $C_{p}$. So there is no invariant operator defined on $\mathcal{D}_{\lambda, \mu}^{0}$.

The last value we will consider in this section is $\nu=\mu-\lambda+p-3$. In this case, we obtain the following classification of the invariant mappings.

Theorem 3.4. - If $p>3$, there exists a one-dimensional space of invariant operators from $\mathcal{D}_{\lambda, \mu}^{p}$ to $\mathcal{F}_{\mu-\lambda+p-3}$ for $(\lambda, \mu)$ equal to the points

$$
\left(\lambda_{0}, \mu_{0}\right)=\left(\frac{2 p-6+\sqrt{p(p-3)}}{6}, \frac{-2 p-\sqrt{p(p-3)}}{6}\right)
$$

or

$$
\left(\lambda_{0}^{\prime}, \mu_{0}^{\prime}\right)=\left(\frac{2 p-6-\sqrt{p(p-3)}}{6}, \frac{-2 p+\sqrt{p(p-3)}}{6}\right) .
$$

- If $p=3$, the spaces of invariant mappings are generated by $\mathcal{P}_{0}$ on the line of equation $\lambda=0$ and by $\mathcal{P}_{0} \circ \mathcal{C}$ on the line of equation $\mu=-1$.

- If $p=2$, there exists solutions for $\lambda=\mu=0$ or $\lambda=\mu=-1$, namely the scalar multiples of $d \circ \mathcal{P}_{0}$ and $d \circ \mathcal{P}_{0} \circ \mathcal{C}$.

Proof. If $p \geqslant 3$, we write $T_{p}=C_{p} \eta^{3}, T_{p-1}=C_{p-1} \eta^{2}, T_{p-2}=C_{p-2} \eta, T_{p-3}=C_{p-3}$ and $T_{r}=0$ for every $r \in\{0, \ldots, p-4\}$. Moreover, the coefficients are submitted to the following conditions.

$$
\left\{\begin{array}{lll}
6(\lambda-\mu-p+1) C_{p}+p(2 \lambda-p+1) C_{p-1} & =0 \\
2(2 \lambda-2 \mu-2 p+3) C_{p-1}+(p-1)(2 \lambda-p+2) C_{p-2} & =0 \\
2(\lambda-\mu-p+2) C_{p-2}+(p-2)(2 \lambda-p+3) C_{p-3} & = & 0 \\
6(3 \lambda-3 \mu-3 p+1) C_{p}+6 p(2 \lambda-p+1) C_{p-1} & \\
+p(p-1)(3 \lambda-p+2) C_{p-2} & =0 \\
6(\lambda-\mu-p+1) C_{p-1}+3(p-1)(2 \lambda-p+2) C_{p-2} & \\
+(p-1)(p-2)(3 \lambda-p+3) C_{p-3} & =0
\end{array}\right.
$$

We compute the rank of the system and get the conditions on the parameters $\lambda$ and $\mu$ to have a solution. This also yields the conditions on the parameters and the dimension of the space of solutions for $p=2$ and $p=3$.

We can explain the values $\left(\lambda_{0}, \mu_{0}\right)$ and $\left(\lambda_{0}^{\prime}, \mu_{0}^{\prime}\right)$ : these are the intersections of the Hyperbola $H_{p-1}$ and the line of equation $\lambda+\mu+1=0$. The interpretation of the invariant mappings in this case is also clear : they are the scalar multiples of the composition $\mathcal{T}_{p-1,2} \circ \mathcal{P}_{\mathcal{C}}$.

3.5. Invariant mappings for higher values of $s$. We will classify the invariant mappings for the remaining cases. First we have to define some new invariants.

Definition 3.4. We denote by $\delta$ the operator of right composition with the de Rham differential d :

$$
\delta: \mathcal{D}_{-1, \mu}^{p} \rightarrow \mathcal{D}_{0, \mu}^{p+1}: D \mapsto D \circ d .
$$

The map $\delta$ is obviously invariant. Moreover it has values in the space of operators vanishing on the constants $\mathcal{D}_{0, \mu, \mathrm{nc}}^{p+1}$. It is also easily seen that the mapping $\delta$ provides an isomorphism between the considered spaces. This allows us to set the following definition. 
Definition 3.5. The mapping $\mathcal{P}_{1}$ from $\mathcal{D}_{0,-1}^{p}$ onto $\mathcal{F}_{0}$ is defined by

$$
\mathcal{P}_{1}: \mathcal{D}_{0,-1}^{p} \rightarrow \mathcal{F}_{0}: \mathcal{P}_{0} \circ \mathcal{C} \circ \delta^{-1} \circ\left(\mathrm{Id}-\mathcal{P}_{0}\right) .
$$

Now, the next theorem ends our study of the invariant mappings from $\mathcal{D}_{\lambda, \mu}^{p}$ to $\mathcal{F}_{\nu}$.

Theorem 3.5. The space of invariant mappings from $\mathcal{D}_{\lambda, \mu}^{p}$ onto $\mathcal{F}_{\mu-\lambda+p-a}$ with $a \geqslant 4$ is generated by $\mathcal{P}_{0}, d \circ \mathcal{P}_{0}, \mathcal{P}_{0} \circ \mathcal{C}, d \circ \mathcal{P}_{0} \circ \mathcal{C}$ and $\mathcal{P}_{1}$.

Proof. Here we will suppose that $s=-p+a$ for an integer $a \geqslant 4$. First we will suppose in addition that $s \leqslant 0$. So we can write

$$
T=\sum_{r=p-a}^{p} T_{r}=\sum_{r=p-a}^{p} C_{r} \eta^{r+a-p} .
$$

We translate the invariance equations in this context and get

$$
\begin{cases}(r+a-p)(2 \lambda-2 \mu+a-p-r-1) C_{r}+r(2 \lambda-r+1) C_{r-1} & =0 \\ (r+a-p)(r+a-p-1)(3 \lambda-3 \mu-2 r+a-p-2) C_{r} & \\ +3 r(2 \lambda-r+1)(r+a-p-1) C_{r-1}+r(r-1)(3 \lambda-r+2) C_{r-2} & =0 .\end{cases}
$$

We use the first equation with $C_{r-1}$ to get

$$
(r+a-p-1)(2 \lambda-2 \mu+a-p-r) C_{r-1}+(r-1)(2 \lambda-r+2) C_{r-2}=0 .
$$

We obtain a system of equations involving $C_{r}, C_{r-1}$ and $C_{r-2}$, for every $r \in$ $\{p-a+2, \ldots, p\}$ (note that there are at least three values of $r$ in this set). We compute the determinant of this system and get a polynomial of degree six in $r$, which can be written

$$
P(r)=(r+a-p)(r+a-p-1) r(r-1) P^{\prime}(r),
$$

where $P^{\prime}$ is a polynomial of degree two in $r$ involving all the parameters. Following our hypotheses, the zeros of $P$ are the ones of $P^{\prime}$. If $P^{\prime}$ is not vanishing identically, then there exists at least one value $r_{0}$ such that $P\left(r_{0}\right) \neq 0$ and so $C_{r_{0}}=C_{r_{0}-1}=$ $C_{r_{0}-2}=0$. We then consider the coefficients of $C_{r}$ in the equations and find that they vanish simultaneously if and only if $r=p-a-1$. This case is not allowed by our hypotheses. So we have $C_{r}=0$ for all $r \geqslant r_{0}$.

We do the same with the coefficients of $C_{r-2}$ and find that they vanish simultaneously if and only if $r=2$ and $\lambda=0$. In this case, we can have a one-dimensional space of solutions, corresponding to the coefficient $C_{0}$. Note that the existence of such solutions requires the existence of $C_{0}$. Hence, we must have $s \geqslant 0$, that is, in our case, $s=0$.

Now we have to analyze the solutions when the polynomial $P^{\prime}(r)$ vanishes identically, that is, when

$$
\begin{aligned}
& -p=a \text { and } \mu=-1, \\
& \text { - } p=a+1, \lambda=0 \text { and } \mu=-1, \\
& \text { - } p=a-1, \lambda=-1 \text { and } \mu=-1 \text {. }
\end{aligned}
$$

It is easily seen that the first case admits a one-dimensional space of solutions if $\lambda \neq 0$ (the solutions being multiples of $\mathcal{P}_{0} \circ \mathcal{C}$ ), and that the space of solutions has dimension two if $\lambda=0$ (the additional generator being $\mathcal{P}_{0}$ ). For the second case, we have a one-dimensional space of solutions generated by $\mathcal{P}_{1}$, and the third case corresponds to a positive value of $s$. 
If $s$ is strictly positive, we write

$$
T=\sum_{r=0}^{p} C_{r} \eta^{r+a-p}
$$

and we have the same system excepts for the values $C_{0}$ and $C_{1}$. There are additional conditions on these constants, anmely

$$
\begin{cases}(2 \lambda-2 \mu+a-p-1) C_{0} & =0 \\ (a-p-1)(3 \lambda-3 \mu-2 r+a-p-2) C_{0} & =0 \\ (a-p+1)(3 \lambda-3 \mu+a-p-4) C_{1}+6 \lambda C_{0} & =0\end{cases}
$$

We also note that the vanishing of $C_{0}$ implies the vanishing of $C_{r}$ for all $r \in$ $\{0, \ldots, p\}$, since the coefficients of $C_{r}$ do not vanish simultaneously in the equations. Now, the system above implies $C_{0}=0$ except in the case $\lambda=\mu$ and $p=a-1$.

In this case, we can consider the systems involving $C_{r}, C_{r-1}$ and $C_{r-2}$ for $r \in$ $\{2, \ldots, p\}$ (with $p=a-1 \geqslant 3$ ). We compute their determinants and obtain the polynomial

$$
P(r)=r^{2}(r+1)(r-1)^{2}(\mu+1)(2+4 \mu-r) .
$$

If $\mu \neq-1$ we can conclude (as in the case $s \leqslant 0$ ) that there exists a one-dimensional space of solutions only if $\lambda=\mu=0$ (a generator being $d \circ \mathcal{P}_{0}: \mathcal{D}_{0,0}^{p} \rightarrow \mathcal{F}_{-1}$ ). If $\lambda=\mu=-1$, we have a one-dimensional space of solutions generated by $d \circ \mathcal{P}_{0} \circ \mathcal{C}$, since the modules $\mathcal{D}_{0,0}^{p}$ and $\mathcal{D}_{-1,-1}^{p}$ are isomorphic by the conjugation.

Now we will present a table that summarizes the results of this section. The first three columns give the conditions on $\nu, \lambda, \mu$ and $p$ to have non trivial solutions,

\begin{tabular}{|c|c|c|c|}
\hline$\nu$ & $p$ & $(\lambda, \mu)$ & Description \\
\hline$\mu-\lambda+p$ & - & - & $\sigma$ \\
\hline \multirow[t]{4}{*}{$\mu-\lambda+p-1$} & \multirow[t]{3}{*}{$\geqslant 1$} & $\neq$ Gel'fand-Dickey & $\mathcal{T}_{p, 1}$ \\
\hline & & \multirow{2}{*}{$=$ Gel'fand-Dickey } & $d \circ \sigma$ \\
\hline & & & $\sigma \circ \mathcal{P}_{\mathcal{C}}$ \\
\hline & $=0$ & $\lambda=\mu$ & $\mathrm{d}$ \\
\hline \multirow[t]{5}{*}{$\mu-\lambda+p-2$} & \multirow[t]{2}{*}{$\geqslant 3$} & $\in H_{p}$ & $\mathcal{T}_{p, 2}$ \\
\hline & & $\mu-\lambda+p-1=0$ & $d \circ \mathcal{T}_{p, 1}$ \\
\hline & \multirow[t]{2}{*}{2} & $\lambda=0$ & $\mathcal{P}_{0}$ \\
\hline & & $\mu=-1$ & $\mathcal{P}_{0} \circ \mathcal{C}$ \\
\hline & 1 & $\lambda-\mu=0$ & $d \circ \mathcal{T}_{p, 1}$ \\
\hline \multirow[t]{3}{*}{$\mu-\lambda+p-3$} & $\geqslant 4$ & $\left(\lambda_{0}, \mu_{0}\right)$ or $\left(\lambda_{0}^{\prime}, \mu_{0}^{\prime}\right)$ & $\mathcal{T}_{p-1,2} \circ \mathcal{P}_{\mathcal{C}}$ \\
\hline & \multirow[t]{2}{*}{3} & $\lambda=0$ & $\mathcal{P}_{0}$ \\
\hline & & $\mu=-1$ & $\mathcal{P}_{0} \circ \mathcal{C}$ \\
\hline \multirow[t]{2}{*}{$\mu-\lambda$} & \multirow[t]{2}{*}{ - } & $\lambda=0$ & $\mathcal{P}_{0}$ \\
\hline & & $\mu=-1$ & $\mathcal{P}_{0} \circ \mathcal{C}$ \\
\hline \multirow[t]{2}{*}{-1} & \multirow[t]{2}{*}{$\geqslant 2$} & $\lambda=\mu=0$ & $d \circ \mathcal{P}_{0}$ \\
\hline & & $\lambda=\mu=-1$ & $d \circ \mathcal{P}_{0} \circ \mathcal{C}$ \\
\hline 0 & $\geqslant 1$ & $(0,-1)$ & $\mathcal{P}_{1}$ \\
\hline
\end{tabular}
the fourth one gives an intrinsic interpretation of the generators of the space of solutions when it is possible. 


\section{NON LOCAL MAPPINGS}

In this section, we will compute all the invariant mappings from $\mathcal{D}_{\lambda, \mu}^{p}$ to $\mathcal{F}_{\nu}$ that do not preserve the support of their arguments, over a manifold of dimension one. The method to find these mappings was already used in [11]. It is based on a result of [2] and we recall here some definitions of that paper.

Definition 4.1. Let $E(M)$ be a vector bundle over a manifold $M$. A family $\mathcal{L}$ of differential operators from $\Gamma(E(M))$ into $\Gamma(E(M))$ is globally transitive if for every open subset $\omega$ of $M$ and every section $s$ with support in $\omega, s$ can be written

$$
s=\sum_{i=1}^{k} L_{i} s_{i},
$$

with $L_{i} \in \mathcal{L}, s_{i} \in \Gamma(E(M))$ and supp $L_{i}$, supp $s_{i} \subset \omega$.

Definition 4.2. A family $\mathcal{L}$ of differential operators from $\Gamma(E(M))$ into $\Gamma(E(M))$ is locally transitive if each point $x$ of $M$ has a neighborhood $\Omega$ such that (4) holds true for every subset $\omega$ of $\Omega$ and every s with compact support in $\omega$, the number of terms in (4) being bounded above independently of $s$ and $\Omega$.

Now we come to our new definition (generalizing property (1) of Section 3 in [11]).

Definition 4.3. Let $E(M)$ be a vector bundle over a manifold $M$. Let $\mathcal{L}$ be a family of differential operators from $\Gamma(E(M))$ into $\Gamma(E(M))$. The space $\Gamma(E(M))$ admits the decomposition property for $\mathcal{L}$ if for every section $s$ and every point $x \in M \backslash$ supp $s$, there exists $L_{i} \in \mathcal{L}, s_{i} \in \Gamma(E(M)),(i \leqslant k)$ such that $x \notin$ supp $L_{i}$ and

$$
s=\sum_{i=1}^{k} L_{i} s_{i} .
$$

Of course we have the following result.

Lemma 4.1. Global transitivity implies the decomposition property.

Moreover, in [2], M. De Wilde and P.B.A Lecomte have proved

Lemma 4.2. If the family $\mathcal{L}$ of differential operators is stable for locally finite sums, then local transitivity for $\mathcal{L}$ implies global transitivity for $\mathcal{L}$.

Now, we will show how one can adapt the results of [11] to the case of a manifold of dimension one. First we have the analogue of Lemma 3.1 of that paper :

Lemma 4.3. For any $r$ and $\nu$ such that $r+\nu+1 \neq 0$, the family of Lie derivatives is locally transitive on the vector bundle $\vee^{r} T M \otimes \Delta^{-\nu}(M)$.

Proof. The reader will easily convince himself that it is sufficient to show that any section of the bundle $V^{r} T \mathbb{R} \otimes \Delta^{-\nu}(\mathbb{R})$ with compact support in $\mathbb{R}$ can be decomposed as a finite sum of Lie derivatives of sections with compact support in $\mathbb{R}$ along vector fields having also compact support (the number of terms being bounded independently of the section).

So, let $u$ be a smooth section of $\vee^{r} T \mathbb{R} \otimes \Delta^{-\nu}(\mathbb{R})$, with compact support in $\mathbb{R}$. We can write

$$
u=f|d t|^{-\nu} \otimes D_{t}^{r}
$$


where $f \in C^{\infty}(\mathbb{R})$ has compact support. We have

$$
L_{t D_{t}}\left(f|d t|^{-\nu} \otimes D_{t}^{r}\right)=\left(t D_{t} f\right)|d t|^{-\nu} \otimes D_{t}^{r}-(\nu+r) f|d t|^{-\nu} \otimes D_{t}^{r}
$$

and

So,

$$
\int_{\mathbb{R}} t D_{t} f-(\nu+r) f d t=-(\nu+r+1) \int_{\mathbb{R}} f d t .
$$

$$
f|d t|^{-\nu} \otimes D_{t}^{r}+\frac{1}{\nu+r+1} L_{t D_{t}}\left(f|d t|^{-\nu} \otimes D_{t}^{r}\right)=\alpha|d t|^{-\nu} \otimes D_{t}^{r}
$$

where $\alpha \in C^{\infty}(\mathbb{R})$ has compact support in $\mathbb{R}$ and $\int_{\mathbb{R}} \alpha d t=0$. Hence we can write $\alpha=D_{t} \beta$ for some $\beta \in C^{\infty}(\mathbb{R})$ with compact support in $\mathbb{R}$.

Finally, we get

$$
f|d t|^{-\nu} \otimes D_{t}^{r}=-\frac{1}{\nu+r+1} L_{\gamma t D_{t}}\left(f|d t|^{-\nu} \otimes D_{t}^{r}\right)+L_{\gamma D_{t}}\left(\beta|d t|^{-\nu} \otimes D_{t}^{r}\right),
$$

if $\gamma \in C^{\infty}(\mathbb{R})$ has compact support in $\mathbb{R}$ and is equal to one on $\operatorname{supp} f \cup \operatorname{supp} \beta$.

Remark : The remaining spaces $\Gamma\left(\vee^{r} T M \otimes \Delta^{r+1}(M)\right)$ are isomorphic to $\mathcal{F}_{-1}$. The natural non local operator in this context is the integral, at least when the manifold is compact.

Now we obtain the analogue of Lemma 3.2 of [11] for the modules of differential operators.

Theorem 4.1. The family of Lie derivatives is globally transitive on the modules $\mathcal{D}_{\lambda, \mu}^{p}(M)$ except $\mathcal{D}_{0,-1}^{p}(M), \mathcal{D}_{\lambda, \lambda-p-1}^{p}$, and $\mathcal{D}_{\frac{p-1}{2}, \frac{-p-1}{2}}^{p}(M)$. Moreover for each of these three modules, there exists a surjective invariant application $\mathcal{A}$ with values in $\mathcal{F}_{-1}$ such that the family of Lie derivatives is globally transitive on the kernel of $\mathcal{A}$.

Proof. Consider a module $\mathcal{D}_{\lambda, \mu}^{p}(M)$. If $\mu-\lambda+r+1 \neq 0$ for every $r \in\{0, \ldots, p\}$, we conclude as in [11], applying Lemma 4.3.

Now, we have to consider the case $\mu-\lambda+r+1=0$ for some $r \in\{0, \ldots, p\}$. If $D \in \mathcal{D}_{\lambda, \mu}^{p}$, we can write

$$
D=|d t|^{\lambda-\mu} \otimes\left(f_{p} D_{t}^{p}+\cdots+f_{r} D_{t}^{r}+\cdots\right) .
$$

If $r=p$, then we have $\mu=\lambda-p-1$. The invariant application with values in $\mathcal{F}_{-1}$ is given by $\sigma$.

If $r<p$, we can try to remove the "singular" term $f_{r}$ by adding a Lie derivative acting on an operator of order $r+1$. We consider the following expressions :

$$
\left.L_{t^{2} D_{t}}\left(f_{r}|d t|^{\lambda-\mu} \otimes D_{t}^{r+1}\right)\right|_{r}=(r+1)(2 \lambda-r)\left(f_{r}|d t|^{\lambda-\mu} \otimes D_{t}^{r}\right)
$$

and

$$
\left.L_{t^{3} D_{t}}\left(f_{r}|d t|^{\lambda-\mu} \otimes D_{t}^{r+2}\right)\right|_{r}=(r+2)(r+1)(3 \lambda-r)\left(f_{r}|d t|^{\lambda-\mu} \otimes D_{t}^{r}\right) .
$$

Note that the second one is only useful if $r<p-1$. These expressions allow us to remove the singular term except if $r=p-1$ and $2 \lambda-r=0$ and if $\lambda=0$ and $r=0$. Taking into account the relation $\mu-\lambda+r+1=0$, this leads to the modules $\mathcal{D}_{\frac{p-1}{2}, \frac{-p-1}{2}}^{p}(M)$ and $\mathcal{D}_{0,-1}^{p}(M)$ respectively. For the first one, the invariant application with values in $\mathcal{F}_{-1}$ is given by $\sigma \circ \mathcal{P}_{\mathcal{C}}+\frac{p}{2} d \circ \sigma$ and for the second one, it is given by $\mathcal{P}_{0}$.

Now, as in [11], we analyze the decomposition property for $\mathcal{F}_{-1}(M)$, for a manifold of dimension one. 
Lemma 4.4. - The space $\mathcal{F}_{-1}(\mathbb{R})$ admits the decomposition property for the family of Lie derivatives.

- For the space $\mathcal{F}_{-1}\left(S^{1}\right)$, the kernel of the integral mapping admits the decomposition property, that is, a section $u \in \mathcal{F}_{-1}\left(S^{1}\right)$ admits a decomposition if and only if $\int_{S^{1}} u=0$.

Proof. Let $x$ be a point of $S^{1}$. The space $S^{1} \backslash\{x\}$ is diffeomorphic to $\mathbb{R}$ and the sections of $\mathcal{F}_{-1}\left(S^{1}\right)$ with support in $S^{1} \backslash\{x\}$ correspond to sections with compact support in $\mathbb{R}$. Hence, we can apply Lemma 3.5 of [11] to obtain the result.

If $x$ is a point of $\mathbb{R}$ and $u \in \mathcal{F}_{-1}(\mathbb{R})$ has support in $\mathbb{R} \backslash\{x\}$, then we can apply a straightforward adaptation of Lemma's 3.3 and 3.6 of [11] to $\left.u\right|_{]-\infty, x[}$ and to $\left.u\right|_{] x,+\infty[}$ to get the result.

Now, we can characterize the non local invariants.

Theorem 4.2. Every invariant operator from the space $\mathcal{D}_{\lambda, \mu}^{p}(\mathbb{R})$ to $\mathcal{F}_{\nu}(\mathbb{R})$ is local The non local invariant operators defined on the modules $\mathcal{D}_{\lambda, \mu}^{p}\left(S^{1}\right)$ are scalar multiples of the following ones.

- $\mathcal{T}_{n l, 1}: \mathcal{D}_{\lambda, \lambda-p-1}^{p}\left(S^{1}\right) \rightarrow \mathbb{R}: D \mapsto \int_{S^{1}} \sigma(D)$,

- $\mathcal{T}_{n l, 2}: \mathcal{D}_{\frac{p-1}{2}, \frac{-p-1}{2}}^{p}\left(S^{1}\right) \rightarrow \mathbb{R}: D \mapsto \int_{S^{1}} \sigma \circ \mathcal{P}_{\mathcal{C}}(D)$,

- $\mathcal{T}_{n l, 3}: \mathcal{D}_{0,-1}^{p^{2}}\left(S^{1}\right) \rightarrow \mathbb{R}: D \mapsto \int_{S^{1}} \mathcal{P}_{0}(D)$.

Proof. The case of invariant operators defined over $\mathbb{R}$ is obvious since the modules $\mathcal{D}_{\lambda, \mu}^{p}(\mathbb{R})$ admit the decomposition property. So is it for the modules $\mathcal{D}_{\lambda, \mu}^{p}\left(S^{1}\right)$, with $(\lambda, \mu)$ such that $\mu \neq \lambda-p-1,(\lambda, \mu) \neq\left(\frac{p-1}{2}, \frac{-p-1}{2}\right)$ and $(\lambda, \mu) \neq(0,-1)$.

Now, let $\mathcal{D}_{\lambda, \mu}^{p}\left(S^{1}\right)$ be one of the remaining modules. Let $\mathcal{T}$ be an invariant mapping defined on $\mathcal{D}_{\lambda, \mu}^{p}$ with values in $\mathcal{F}_{\nu}, D \in \mathcal{D}_{\lambda, \mu}^{p}$, and $x \in S^{1} \backslash \operatorname{supp}(D)$. Using the notation of Theorem 4.1, it is obvious that the image $(\mathcal{T}(D))_{x}$ only depends on the values

$$
\int_{S^{1}} \mathcal{A}(D)
$$

Moreover, we have, for any $X \in \operatorname{Vect}\left(S^{1}\right)$

$$
\left(L_{X} \mathcal{T}(D)\right)_{x}=\left(\mathcal{T}\left(L_{X} D\right)\right)_{x}=0,
$$

since

$$
\int_{S^{1}} \mathcal{A}\left(L_{X} D\right)=\int_{S^{1}} L_{X} \mathcal{A}(D)=0
$$

Hence, we have $\nu=0$ and $\mathcal{T}(D)$ is a locally constant function (outside the support of $D$ ), that only depends on $\int_{S^{1}} \mathcal{A}(D)$.

\section{Some Submodules}

Here we will give some examples of submodules of the spaces $\mathcal{D}_{\lambda, \mu}^{p}$ that one can construct using the invariant mappings we have listed above, especially over a manifold of dimension one.

Some of them are well-known : let us quote the kernel of $\sigma$, which corresponds to the submodule of operators of order at most $p-1$, or the kernel of $\mathcal{P}_{0}$, when $\lambda=0$, which is constituted of operators vanishing on the constants. But we can present some new submodules. 
5.1. Submoduloes associated to $\mathcal{T}_{p, 1}$. In the general situation, considering the kernel of $\mathcal{T}_{p, 1}$ leads to the operators of the form

$$
f_{p} D_{t}^{p}+\frac{p(2 \lambda-p+1)}{2(\lambda-\mu-p)} D_{t} f_{p} D_{t}^{p-1}+\sum_{k=0}^{p-2} f_{k} D_{t}^{k}
$$

When $\mu-\lambda+p=0 \mathcal{T}_{p, 1}$ becomes $d \circ \sigma$ and the corresponding submodule is constituted by the operators with constant principal symbol :

$$
c D_{t}^{p}++\sum_{k=0}^{p-1} f_{k} D_{t}^{k}, \quad c \in \mathbb{R}
$$

For $\lambda=\frac{p-1}{2}$, the general case degenerates and we obtain operators of the form

$$
f_{p} D_{t}^{p}+\sum_{k=0}^{p-2} f_{k} D_{t}^{k}
$$

The module $\mathcal{D}_{\frac{p-1}{2}, \frac{-p-1}{2}}^{p}$ is at the intersection of the lines of equations $\mu-\lambda+p=0$ and $\lambda=\frac{p-1}{2}$, hence we can consider in this situation the submodule of operators

$$
c D_{t}^{p}+\sum_{k=0}^{p-2} f_{k} D_{t}^{k}, \quad c \in \mathbb{R}
$$

5.2. Submodules associated to $\mathcal{T}_{p, 2}$. The mapping $\mathcal{T}_{p, 2}$ is defined on the modules $\mathcal{D}_{\lambda, \mu}^{p}$ with $(\lambda, \mu)$ belonging to the hyperbola $H_{p}(p \geqslant 3)$. Using the notation of Section 3.4 , it is easily seen that the coefficient $C_{p-2}$ cannot vanish and we can consider operators of the form

$$
f_{p} D_{t}^{p}+f_{p-1} D_{t}^{p-1}-\left(\frac{C_{p}}{C_{p-2}} D_{t}^{2} f_{p}+\frac{C_{p-1}}{C_{p-2}} D_{t} f_{p-1}\right) D_{t}^{p-2}+\sum_{k=0}^{p-3} f_{k} D_{t}^{k} .
$$

We can also consider the intersections of the hyperbola $H_{p}$ with the line of equation $\mu-\lambda+p=0$ and we obtain the modules $\mathcal{D}_{\lambda, \mu}^{p}$ with

$$
(\lambda, \mu)=\left(\frac{3 p-3-\sqrt{3(p+1)}}{6}, \frac{-3-3 p-\sqrt{3(p+1)}}{6}\right),
$$

or

$$
(\lambda, \mu)=\left(\frac{3 p-3+\sqrt{3(p+1)}}{6}, \frac{-3-3 p+\sqrt{3(p+1)}}{6}\right) .
$$

In these modules, one can consider the submodules of the operators of the form

$$
c D_{t}^{p}+f_{p-1} D_{t}^{p-1}-\frac{C_{p-1}}{C_{p-2}} D_{t} f_{p-1} D_{t}^{p-2}+\sum_{k=0}^{p-3} f_{k} D_{t}^{k}, \quad c \in \mathbb{R} .
$$

Some of these submodules have been considered in [1] for $p=2$ and $p=3$. 
5.3. Weak form of the "Gel'fand-Dickey" submodule. If we consider the non local mapping defined on $\mathcal{D}_{\frac{p-1}{2}, \frac{p-1}{2}}^{p}\left(S^{1}\right)$, we obtain the submodule of operators of the form

$$
c D_{t}^{p}+f_{p-1} D_{t}^{p-1}+\sum_{k=0}^{p-2} f_{k} D_{t}^{k},
$$

with the condition

$$
\int_{S^{1}} f_{p-1}=0
$$

This submodule can be considered as a weak form of the "Gel'fand-Dickey" submodule that we presented in the introduction.

\section{REFERENCES}

[1] S. Bouarroudj and V. Ovsienko. Three cocycles on Diff $\left(S^{1}\right)$ generalizing the Schwarzian derivative. Internat. Math. Res. Notices, (1):25-39, 1998.

[2] M. De Wilde and P. Lecomte. Some characterizations of differential operators on vector bundles. In E. B. Christoffel (Aachen/Monschau, 1979), pages 543-549. Birkhäuser, Basel, 1981.

[3] C. Duval and V. Ovsienko. Space of second-order linear differential operators as a module over the Lie algebra of vector fields. Adv. Math., 132(2):316-333, 1997.

[4] D. B. Fuks. Cohomology of infinite-dimensional Lie algebras. Contemporary Soviet Mathematics. Consultants Bureau, New York, 1986. Translated from the Russian by A. B. Sosinski1.

[5] H. Gargoubi. Sur la géométrie de l'espace des opérateurs différentiels linéaires sur R. Bull. Soc. Roy. Sci. Liège, 69(1):21-47, 2000.

[6] H. Gargoubi and V. Ovsienko. Space of linear differential operators on the real line as a module over the Lie algebra of vector fields. Internat. Math. Res. Notices, (5):235-251, 1996.

[7] A. A. Kirillov. Infinite-dimensional Lie groups: their orbits, invariants and representations. The geometry of moments. In Twistor geometry and nonlinear systems (Primorsko, 1980), volume 970 of Lecture Notes in Math., pages 101-123. Springer, Berlin, 1982.

[8] P. B. A. Lecomte. On the cohomology of $\mathrm{sl}(m+1, \mathbb{R})$ acting on differential operators and $\operatorname{sl}(m+1, \mathbb{R})$-equivariant symbol. Indag. Math. (N.S.), 11(1):95-114, 2000.

[9] P. B. A. Lecomte, P. Mathonet, and E. Tousset. Comparison of some modules of the Lie algebra of vector fields. Indag. Math. (N.S.), 7(4):461-471, 1996.

[10] P. B. A. Lecomte and V. Ovsienko. Projectively equivariant symbol calculus. Lett. Math. Phys., 49(3):173-196, 1999.

[11] P. Mathonet. Intertwining operators between some spaces of differential operators on a manifold. Comm. Algebra, 27(2):755-776, 1999.

[12] J. Peetre. Une caractérisation abstraite des opérateurs différentiels. Math. Scand., 7:211-218, 1959.

[13] L. Schwartz. Théorie des distributions. Publications de l'Institut de Mathématique de l'Université de Strasbourg, No. IX-X. Nouvelle édition, entiérement corrigée, refondue et augmentée. Hermann, Paris, 1966.

[14] G. Segal. Unitary representations of some infinite-dimensional groups. Comm. Math. Phys., 80(3):301-342, 1981.

[15] H. Weyl. The classical groups. Princeton Landmarks in Mathematics. Princeton University Press, Princeton, NJ, 1997. Their invariants and representations, Fifteenth printing, Princeton Paperbacks.

[16] E. J. Wilczynski. Projective differential geometry of curves and ruled surfaces. LeipzigTeubner, 1906.

(Pierre Mathonet) University of Liège, Department of Mathematics, Grande Traverse, 12 - B37, B-4000 LiÈGe, BELGIUM

E-mail address: P.Mathonet [at]ulg.ac.be 